Cahiers $d u$ MONDE RUSSE

\section{Cahiers du monde russe}

Russie - Empire russe - Union soviétique et États indépendants

$51 / 4 \mid 2010$

Sciences humaines et sociales en Russie à l'Âge d'argent

\title{
Boris Makarov, Gollandcy v Rossii v pervoj polovine xviii veka
}

Dmitri Gouzévitch

\section{(2) OpenEdition \\ Journals}

Édition électronique

URL : https://journals.openedition.org/monderusse/7367

DOI : $10.4000 /$ monderusse. 7367

ISSN : $1777-5388$

Éditeur

Éditions de l'EHESS

\section{Édition imprimée}

Date de publication : 25 novembre 2010

Pagination : 675-678

ISBN : 978-2-7132-2316-7

ISSN : $1252-6576$

Référence électronique

Dmitri Gouzévitch, «Boris Makarov, Gollandcy v Rossii v pervoj polovine xviii veka», Cahiers du monde russe [Онлайн], 51/4 | 2010, Выложить онлайн 09 septembre 2013, Наводить справки в 03 septembre 2022. URL: http://journals.openedition.org/monderusse/7367 ; DOI: https://doi.org/ $10.4000 /$ monderusse. 7367

Ce document a été généré automatiquement le 3 septembre 2022. 


\title{
Boris Makarov, Gollandcy v Rossii v pervoj polovine xviii veka
}

\author{
Dmitri Gouzévitch
}

\section{RÉFÉRENCE}

Boris MAKAROV, Gollandcy v Rossii v pervoj polovine XvIII veka : Leksikon [pred. E.

Vagemans] [Boris Makarov, Les Hollandais en Russie dans la première moitié du XVIII ${ }^{e}$ siècle : Lexique, introd. de E. Vagemans]. Saint-Pétersbourg : NRC, 2009, 283 p.

1 КНИГА РОДИЛАСЬ КАК РЕЗУЛЬТАТ ГОЛЛАНДСКО-РОССИЙСКОЙ КООПЕРАЦИИ И МНОГОЛЕТНЕГО ПОДВИЖНИЧЕСКОГО ТРУДА АВТОРА. РАБОТА, БЕЗУСЛОВНО, НОСИТ ПИОНЕРНЫЙ ХАРАКТЕР. И УЖЕ СТАЛА РЕФЕРЕНТНОЙ.

ОСНОВНЫМ ЕЕ ДОСТОИНСТВОМ ЯВЛЯЕТСЯ ВВЕДЕНИЕ В НАУЧНЫЙ ОБОРОТ ОГРОМНОГО ЧИСЛА ДОКУМЕНТОВ, СИСТЕМАТИЗИРОВАННЫХ ПО ПЕРСОНАЛИЯМ. ПРИЧЕМ ЦЕННОСТЬ ЭТОЙ РАБОТЫ НЕ СТОЛЬКО В ОТКРЫТИИ ДЛЯ ИССЛЕДОВАТЕЛЕЙ БОЛЬШОГО ЧИСЛА НОВЫХ АРХИВНЫХ ДЕЛ, СКОЛЬКО СБОР И СИСТЕМАТИЗАЦИЯ КРУПИЦ, РАЗБРОСАННЫХ ПО ТЫСЯЧАМ АРХИВНЫХ СТРАНИЦ. ИХ СОЕДИНЕНИЕ В РАМКАХ СЛОВАРЯ ПОЗВОЛЯЕТ СОЗДАТЬ ЦЕЛОСТНУЮ КАРТИНУ.

НАША ДАЛЬНЕЙШАЯ ОЦЕНКА ДОЛЖНА ЗАВИСЕТЬ ОТ ПРИНЦИПИАЛЬНОЙ ПОЗИЦИИ: СЧИТАЕМ ЛИ МЫ ЭТОТ ЛЕКСИКОН ЗАКОНЧЕННЫМ ИССЛЕДОВАНИЕМ ИЛИ ЛИШЬ ПЕРВЫМ ШАГОМ ПО ПУТИ, КОТОРЫЙ ЕЩЕ ПРЕДСТОИТ ПРОЙТИ АВТОРУ, ЧТОБЫ СОЗДАТЬ ЗАВЕРШЕННЫЙ ТРУД ? МЫ ВЫБИРАЕМ ВТОРОЙ ПОДХОД, КОТОРЫЙ ТРЕБУЕТ УКАЗАНИЯ НА ФАКТОЛОГИЧЕСКИЕ ОШИБКИ И НА ДЕФЕКТЫ, ОСЛОЖНЯЮЩИЕ ПОЛЬЗОВАНИЕ КНИГОЙ, А ВСЕ ОСТАЛЬНОЕ АВТОМАТИЧЕСКИ ОКАЗЫВАЕТСЯ РЕКОМЕНДАЦИЯМИ К ДАЛЬНЕЙШЕМУ РАЗВИТИЮ ИССЛЕДОВАНИЯ.

4 АВТОР - БЛЕСТЯЩИЙ ФАКТОЛОГ И АРХИВИСТ. ОСНОВНЫЕ ЖЕ ВОПРОСЫ К НЕМУ ВОЗНИКАЮТ НА УРОВНЕ ОБОБЩЕНИЯ И АНАЛИЗА, А ТАКЖЕ ИСПОЛЬЗОВАНИЯ 
ИСТОРИОГРАФИИ. НЕ СЛУЧАЙНО ОСНОВНАЯ НАША КРИТИКА ОТНОСИТСЯ К ПРЕДИСЛОВИЮ.

5 ТАК, ОБРАТИМ ВНИМАНИЕ НА УПОМИНАЕМЫЕ В ПРЕДИСЛОВИЯХ ОТ РЕДАКТОРА (С. 4) И АВТОРА (С. 7) УКАЗАНИЯ НА «НЕВЛАДЕНИЕ ИНОСТРАННЫМИ ЯЗЫКАМИ » РУССКИХ КАНЦЕЛЯРИСТОВ, И НА ИХ « ПЛОХОЕ ОБРАЗОВАНИЕ ». ЭТО ПРИВОДИТСЯ В КАЧЕСТВЕ ПРИЧИНЫ ВАРИАТИВНОСТИ ИНОСТРАННЫХ ИМЕН, ФИКСИРОВАВШИХСЯ В РУССКИХ ДОКУМЕНТАХ НА СЛУХ. БЕЗУСЛОВНО, ЭТИ ПРИЧИНЫ ИМЕЛИ МЕСТО, ОДНАКО В ОСНОВЕ ВАРИАТИВНОСТИ ВСЕ-ТАКИ ЛЕЖАТ НЕ ОНИ, А ОТСУТСТВИЕ НОРМАЛИЗАЦИИ. В ЭТУ ЭПОХУ В РОССИИ В ПРИНЦИПЕ ОТСУТСТВОВАЛИ НЕ ТОЛЬКО ЗАКРЕПЛЕННЫЕ НОРМАМИ ИЛИ ХОТЯ БЫ ОБЩЕПРИНЯТЫЕ СИСТЕМЫ ТРАНСЛИТЕРАЦИИ ИНОСТРАННЫХ ИМЕН, НО МЕНЯЛИСЬ КАК САМА КИРИЛЛИЦА, ТАК И РУССКИЙ ПИСЬМЕННЫЙ ЯЗЫК. ВСЕ БЫЛО СЛИШКОМ ТЕКУЧЕ, И БЕДНЫЕ ПОДЪЯЧИЕ И КАНЦЕЛЯРИСТЫ ПРОСТО НЕ ЗНАЛИ, КАК ОНИ ДОЛЖНЫ ПИСАТЬ. ВПРОЧЕМ, В ТУ ЭПОХУ ЭТОГО НЕ ЗНАЛ НИКТО. ЭТА ПРОБЛЕМА НАКЛАДЫВАЛАСЬ НА ВАРИАТИВНОСТЬ ЕВРОПЕЙСКИХ ФОРМ ЭТИХ ЖЕ ИМЕН, ЧТО ОТМЕЧАЕТ АВТОР : «САМИ ИНОСТРАНЦЫ ПОДПИСЫВАЛИСЬ ПО-РАЗНОМУ <... >. В ТАКИХ СЛУЧАЯХ ПРИВОДИЛИСЬ НАИБОЛЕЕ ЧАСТО ВСТРЕЧАЮЩИЕСЯ ВАРИАНТЫ ПОДПИСЕЙ » (С. 10). ДУМАЕМ, ЧТО В ПОДОБНЫХ СЛУЧАЯХ НЕОБХОДИМО ПРИВОДИТЬ ВСЕ ОБНАРУЖЕННЫЕ ВАРИАНТЫ.

6 И ДАЛЕЕ, УКАЗЫВАЯ НА ФАКТЫ ВАРИАТИВНОСТИ, ИСКАЖАЮЩИЕ ИМЕНА ГОЛЛАНДСКИХ И НЕМЕЦКИХ МАСТЕРОВ ПОЧТИ ДО НЕУЗНАВАЕМОСТИ, АВТОР ЗАКЛЮЧАЕТ : « НЕВОЗМОЖНО СОСТАВИТЬ ПОЛНУЮ БИБЛИОГРАФИЮ СО ССЫЛКАМИ НА ВСЕ ПРЕДШЕСТВУЮЩИЕ ИССЛЕДОВАНИЯ » (С. 8). ПРИЧИНЫ НЕВОЗМОЖНОСТИ СОСТАВЛЕНИЯ ПОЛНОЙ БИБЛИОГРАФИИ ИНЫЕ : НИКОГДА НЕЛЬЗЯ БЫТЬ УВЕРЕННЫМ, ЧТО НАЙДЕНЫ ВСЕ ПУБЛИКАЦИИ, ГДЕ УПОМИНАЕТСЯ ИСКОМЫЙ ПЕРСОНАЖ ИЛИ СОБЫТИЕ. А ВАРИАТИВНОСТЬ ИМЕН ПРОСТО ЗАСТАВИТ ПОРАБОТАТЬ ПОБОЛЬШЕ, ПОСТАВИТ НЕКОТОРЫЕ ИЗ ТЕКСТОВ ПОД ВОПРОС И ПОТРЕБУЕТ СОЗДАТЬ СПИСОК НЕИДЕНТИФИЦИРОВАННЫХ ИМЕН, КОТОРЫЕ, ПО МЕРЕ ПОЯВЛЕНИЯ ИНФОРМАЦИИ, БУДУТ РАСШИФРОВЫВАТЬСЯ.

7 ТАКЖЕ МЫ НЕ МОЖЕМ СОГЛАСИТЬСЯ С КАТЕГОРИЧНОСТЬЮ СЛЕДУЮЩЕГО УТВЕРЖДЕНИЯ : « ОПРЕДЕЛЕНИЕ НАЦИОНАЛЬНОСТИ ПО СТРУКТУРЕ ИМЕНИ <... > НЕ ДОСТОВЕРНО » (С. 8). ЕСЛИ БЫ АВТОР БЫЛ ПРАВ, ТО ИССЛЕДОВАНИЯ ПОДОБНОГО РОДА, ВКЛЮЧАЯ И ЕГО СОБСТВЕННОЕ, НЕ ИМЕЛИ БЫ СМЫСЛА. ПОЭТОМУ, НА НАШ ВЗГЛЯД, КОРРЕКТНЕЕ : « ... НЕ ВСЕГДА ДОСТОВЕРНО ». И СТЕПЕНЬ ЭТОЙ ДОСТОВЕРНОСТИ БОЛЕЕ ЛИБО МЕНЕЕ ОПРЕДЕЛЯЕМА. В СОМНИТЕЛЬНЫХ СЛУЧАЯХ ВСЕГДА МОЖНО ДОБАВИТЬ СЛОВА « ПОХОЖЕ », « ВЕРОЯТНО » ИЛИ ПРОСТО ЗНАК ВОПРОСА В СКОБКАХ : «(?) ». ЛЮБАЯ ЖЕ ДОПОЛНИТЕЛЬНАЯ И НЕЗАВИСИМАЯ ИНФОРМАЦИЯ РЕЗКО УВЕЛИЧИВАЕТ ВЕРОЯТНОСТЬ ТОГО ИЛИ ИНОГО РЕШЕНИЯ. ГЛАВНОЕ, ЧТОБЫ ОНА НЕ ОПИРАЛАСЬ НА ФОРМУ ИМЕНИ.

ЧРЕЗВЫЧАЙНО ЛЮБОПЫТНО НАБЛЮДЕНИЕ АВТОРА ПО ПОВОДУ СПИСКОВ МОРЯКОВ, НАНЯТЫХ В 1697-98 ГГ. СПИСКИ ХРАНЯТСЯ В РГАДА, И ДЛЯ БОЛЬШИНСТВА ИМЕН УКАЗАНА НАЦИОНАЛЬНОСТЬ (ПРОИСХОЖДЕНИЕ, ПОДДАНСТВО). НА ОСНОВАНИИ ОРФОГРАФИИ ПОМЕТОК МАКАРОВ ПРИХОДИТ К ВЫВОДУ, ЧТО ОНИ « СДЕЛАНЫ В КОНЦЕ XVIII - ХІХ ВЕКЕ » (С. 9). ПРИВЕДЕННАЯ АРГУМЕНТАЦИЯ УБЕДИТЕЛЬНА, И ВЫВОД ОДНОЗНАЧЕН. НО КАК ТОЛЬКО АВТОР ОТХОДИТ ОТ САМОГО ДОКУМЕНТА, ЕГО ТЕКСТ ВЫЗЫВАЕТ ВОПРОСЫ. ЦИТИРУЕМ : « МОЖНО СДЕЛАТЬ ВЫВОД, ЧТО <... > ЭТА 
ИНФОРМАЦИЯ - РЕЗУЛЬТАТ БОЛЕЕ ПОЗДНЕЙ РАБОТЫ КОГО-ТО ИЗ РУССКИХ ИСТОРИКОВ (?) », И ПЕРЕД ЭТИМ : «ЭТА ИНФОРМАЦИЯ О НАЦИОНАЛЬНОСТИ ИСПОЛЬЗОВАНА В ОГРОМНОМ КОЛИЧЕСТВЕ ПОСЛЕДУЮЩИХ РАБОТ » (С.9) И ДАЕТСЯ ССЫЛКА НА НАШУ РАБОТУ « ПЕРВОЕ ЕВРОПЕЙСКОЕ ПУТЕШЕСТВИЕ ЦАРЯ ПЕТРА: АНАЛИТИЧЕСКАЯ БИБЛИОГРАФИЯ ЗА ТРИ СТОЛЕТИЯ » (СПБ., 2008). МЫ, КОНЕЧНО, ПОЛЬЩЕНЫ, ЧТО НАШ ТРУД ПРИЗНАН В КАЧЕСТВЕ « ОГРОМНОГО КОЛИЧЕСТВА РАБОТ », НО ВСЕ ЖЕ ПОДОБНОЕ ЗАЯВЛЕНИЕ ОБЯЗЫВАЕТ СОСЛАТЬСЯ И НА ДРУГИЕ КНИГИ. И, ЕСЛИ БЫ АВТОР ЭТО СДЕЛАЛ И ПРОШЕЛСЯ ПО ЦЕПОЧКЕ ЦИТИРОВАНИЙ В ОБРАТНУЮ СТОРОНУ, ТО ОБНАРУЖИЛ БЫ, ЧТО ПЕРВЫМ ДАННУЮ ТАБЛИЦУ ОПУБЛИКОВАЛ В КОНЦЕ 1850-Х ГГ. Н.Г. УСТРЯЛОВ (НА КОТОРОГО МАКАРОВ, КСТАТИ, ССЫЛАЕТСЯ НА С. 233). ТАК ОН, СКОРЕЕ ВСЕГО, И ДЕЛАЛ ЭТИ ДОПОЛНЕНИЯ. РУКОПИСИ УСТРЯЛОВА СОХРАНИЛИСЬ, И СРАВНИТЬ ПОЧЕРКА НЕ ТАК СЛОЖНО. Т. О., НЕДОСТАТОЧНОЕ ВНИМАНИЕ К ИСТОРИОГРАФИИ НЕ ДАЛО АВТОРУ ВОЗМОЖНОСТИ ЗАВЕРШИТЬ РЕШЕНИЕ ПРОБЛЕМЫ, КОТОРУЮ ОН САМ ЖЕ И ПОСТАВИЛ.

ВТОР УТВЕРЖДАЕТ, ЧТО ОДНОЙ ИЗ ПРИЧИН БЫСТРОГО ЗАГНИВАНИЯ РУССКИХ КОРАБЛЕЙ « БЫЛА НЕПРАВИЛЬНАЯ ЗАГОТОВКА ЛЕСА » (С. 9). ДУМАЕМ, ЧТО ДЕЛО БЫЛО НЕ ТОЛЬКО В «НЕПРАВИЛЬНОЙ ЗАГОТОВКЕ » (Т.Е. В ЕЕ ТЕХНОЛОГИИ), СКОЛЬКО В СРОКАХ : ПО 1721 Г. ВКЛЮЧИТЕЛЬНО ПЕТР І СТРАШНО ТОРОПИЛСЯ. ВОЙНА ДИКТОВАЛА СВОИ УСЛОВИЯ, И БЕЗ СИЛЬНОГО (ПУСТЬ ДАЖЕ И НЕДОЛГОВЕЧНОГО) РОССИЙСКОГО ФЛОТА ШВЕЦИЯ НЕ ПОДПИСАЛА БЫ МИРНЫЙ ДОГОВОР. К ТОМУ ЖЕ ГОЛЛАНДСКИЕ ТЕХНОЛОГИИ БЫЛИ ИЗВЕСТНЫ. НЕ СЛУЧАЙНО В 1720-ЕГГ. ВОЗНИКЛА « НОВАЯ ГОЛЛАНДИЯ » (НО ТОГДА, ВПОЛНЕ ВОЗМОЖНО, ЧТО ДО НЕЕ БЫЛА ЕЩЕ И СТАРАЯ) СКЛАДЫ ДЛЯ ПРОСУШКИ И ХРАНЕНИЯ ЛЕСА ПО ГОЛЛАНДСКОЙ СИСТЕМЕ. В 1726 Г., КОГДА НАБИРАЛИ НОВЫХ СПЕЦИАЛИСТОВ, СПЕШКА УЖЕ ПРОШЛА, И СТАЛО ВОЗМОЖНЫМ ГОВОРИТЬ О БОЛЕЕ ТЩАТЕЛЬНОМ СОБЛЮДЕНИИ ИЗВЕСТНЫХ РАНЕЕ ТЕХНОЛОГИЙ.

ВЫЗЫВАЕТ НЕДОУМЕНИЕ СЛЕДУЩИЙ ПАССАЖ : «С 1720 ГОДА БЫЛА ВВЕДЕНА ОБЯЗАТЕЛЬНАЯ РЕГИСТРАЦИЯ ПРИЕЗЖАЮЩИХ ИНОСТРАНЦЕВ < ... > (ПРИЛОЖЕНИЕ 7) » (с.11). ВО-ПЕРВЫХ, ИЗ ДОКУМЕНТА, ПРИВЕДЕННОГО В ЭТОМ ПРИЛОЖЕНИИ (С. 19-20), СЛЕДУЕТ, ЧТО РЕЧЬ ИДЕТ НЕ О 1720, А О 1719 Г. С ДРУГОЙ СТОРОНЫ, РЕГИСТРАЦИЯ ПРИЕЗЖАЮЩИХ ИНОСТРАНЦЕВ БЫЛА ОБЯЗАТЕЛЬНОЙ ВСЕГДА. ПРОСТО ПРОИЗВОДИЛИ ЕЕ В МОСКВЕ РАЗНЫЕ ПРИКАЗЫ (ПОСОЛЬСКИЙ, ИНОЗЕМНЫЙ И ДР.). А ЭТОТ УКАЗ - ЛИШЬ ФИКСАЦИЯ ТОГО ФАКТА, ЧТО СОБСТВЕННО РЕГИСТРАЦИЮ СПЕЦИАЛИСТОВ В ПЕТЕРБУРГЕ (КУДА ДВИГАЛСЯ ОСНОВНОЙ ПРОТОК ПРИЕЗЖИХ) И ПОСЛЕДУЮЩЕЕ ИХ НАПРАВЛЕНИЕ ПО ПРИНАДЛЕЖНОСТИ ДОЛЖНА БЫЛА ПРОИЗВОДИТЬ ПОЛИЦИЯ.

11 УТВЕРЖДЕНИЕ АВТОРА, ЧТО НАЧИНАЯ С 1710-Х ГГ. СТАЛА ПРОВОДИТЬСЯ ВЫБОРОЧНАЯ « ЭКЗАМЕНАЦИЯ » ЗАПАДНЫХ СПЕЦИАЛИСТОВ, И ЛИШЬ С 1723 Г. - ТОТАЛЬНАЯ (С. 11), ВЫЗЫВАЕТ СОМНЕНИЕ. ДЕЛО В ТОМ, ЧТО ПРОВЕРКА ПОСТУПАВШИХ НА КОРОННУЮ СЛУЖБУ ПРОВОДИЛАСЬ ИЗДАВНА, ВО ВСЯКОМ СЛУЧАЕ, УЖЕ В ЭПОХУ АЛЕКСЕЯ МИХАЙЛОВИЧА ЭТА ПРАКТИКА БЫЛА МАССОВОЙ. СПЕЦИАЛИСТ ДОЛЖЕН БЫЛ ЛИБО ПРОЙТИ ЭКЗАМЕН (КАК ПЕРЕВОДЧИКИ, ТОЛМАЧИ, АПТЕКАРИ, ВОЕННЫЕ), ЛИБО ПРЕДСТАВИТЬ ДИПЛОМ, АТТЕСТАТ, ПАТЕНТ (БЕЗ ЭТОГО НЕЛЬЗЯ БЫЛО ЗАНЯТЬ ДОЛЖНОСТИ ЛЕКАРЯ, ВРАЧА, ОФИЦЕРА), ХОТЯ ЭКЗАМЕН МОГ БЫТЬ НАЗНАЧЕН И В ЭТОМ СЛУЧАЕ. Т.О., РЕЧЬ ДОЛЖНА ИДТИ НЕ О ТОМ, ЧТО ПЕТР ВВЕЛ ЭКЗАМЕНОВКУ, А О ТОМ, ЧТО В ПЕРИОД МАССОВЫХ НАБОРОВ ЭТО ЖЕСТКОЕ ПРАВИЛО ОСЛАБЛО. И, В РЕЗУЛЬТАТЕ, В 1710-Е ГГ. ПРАВИТЕЛЬСТВО БЫЛО ВЫНУЖДЕНО К НЕМУ ВЕРНУТЬСЯ. С 
ЭТОЙ ЖЕ ТОЧКИ ЗРЕНИЯ НАДО РАССМАТРИВАТЬ И УТВЕРЖДЕНИЕ АВТОРА О ТОМ, ЧТО « ТОЛЬКО К СЕРЕДИНЕ 1710-Х ГОДОВ И САМ ЦАРЬ, И ВЫСШИЕ РУССКИЕ ЧИНОВНИКИ ПРИОБРЕЛИ НЕОБХОДИМЫЕ ЗНАНИЯ И ОПЫТ, ЧТОБЫ ПРОДЕЛАТЬ ЭТУ ВАЖНЕЙШУЮ РАБОТУ » (С. 11). РАНЕЕ ЭКЗАМЕНОВКУ (НАПРИМЕР, АПТЕКАРЕЙ И ВРАЧЕЙ) ПРОВОДИЛА КОМПЕТЕНТНАЯ КОМИССИЯ ИЗ УЖЕ ПРИЗНАНЫХ ИНОСТРАННЫХ МАСТЕРОВ, И ЗНАНИЯ СОБСТВЕННО РУССКИХ ДЛЯ ЭТОГО НЕ ТРЕБОВАЛИСЬ. ПОНЯТНО, ЧТО ПРИ МАССОВОМ НАЙМЕ ТАКАЯ СИСТЕМА ПРОСТО « ЗАХЛЕБНУЛАСЬ ».

ИЗ ТЕКСТА НА С. 13 МОЖНО ПОНЯТЬ, ЧТО АВТОРА ОГРАНИЧИВАЛ ДОПУСТИМЫЙ ОБЪЕМ КНИГИ, И ПОЭТОМУ ДЛЯ ПЕРСОНАЖЕЙ, УПОМИНАЕМЫХ В ОГРОМНОМ КОЛИЧЕСТВЕ ДОКУМЕНТОВ (ТАКИХ, КАК ХАРМАН ВАН БОЛЕС ИЛИ ПИТЕР ВАН ХЕССЕЛЬ), ОН УКАЗЫВАЛ ЛИШЬ ВАЖНЕЙШИЕ ИЗ ЭТИХ ДОКУМЕНТОВ, КАК, ВПРОЧЕМ, И ЛИТЕРАТУРНЫЕ ИСТОЧНИКИ. НУ ЧТО Ж, ЭТУ ПОЗИЦИЮ МОЖНО ПРИНЯТЬ, КАК ТЕХНИЧЕСКИ ВЫНУЖДЕННУЮ, НО ЭТО ЕЩЕ ОДИН ИЗ АРГУМЕНТОВ В ПОЛЬЗУ ТОГО, ЧТО ПЕРЕД НАМИ ПОДГОТОВИТЕЛЬНАЯ, РАБОЧАЯ ВЕРСИЯ ЛЕКСИКОНА. В ОКОНЧАТЕЛЬНОЙ ВЕРСИИ ДОЛЖНЫ БЫТЬ ДАНЫ ССЫЛКИ НА ВСЕ АРХИВНЫЕ ДЕЛА И НА ВСЕ ЛИТЕРАТУРНЫЕ ИСТОЧНИКИ, ВОПРЕКИ УТВЕРЖДЕНИЮ АВТОРА, ЧТО ЭТО НЕЦЕЛЕСООБРАЗНО И УВЕЛИЧИТ « ОБЪЕМ ЛЕКСИКОНА В НЕСКОЛЬКО РАЗ » (С. 13) - ЭТО И БУДЕТ ОДНИМ ИЗ ЕГО БОГАТСТВ.

В ЗАКЛЮЧЕНИЕ, РЯД СООБРАЖЕНИЙ ПО ПРОБЛЕМАМ ПОДБОРА И ПРЕДСТАВЛЕНИЯ ИМЕН, КОТОРЫЕ, БЕЗУСЛОВНО, СУЩЕСТВУЮТ. С ОДНОЙ СТОРОНЫ, ДАН ЖЕСТКИЙ ОРГАНИЧИТЕЛЬ - І ПОЛОВИНА ХVІІ В., А, С ДРУГОЙ, ИМЕНА « РАСПОЛЗАЮТСЯ » И В XVII ВЕК, И ВО II ПОЛОВИНУ ХVIII В. ПРИ ЭТОМ ПОЛНОГО ОХВАТА ЗА ЭТИ ПЕРИОДЫ НЕТ, ЧТО АВТОР САМ ОГОВАРИВАЕТ. НО ВОТ ПРИМЕР. ПАТРИК ГОРДОН БЫЛ ДВАЖДЫ ЖЕНАТ НА ГОЛЛАНДКАХ. ВНАЧАЛЕ - НА КАТАРИНЕ, ДОЧЕРИ ПОЛКОВНИКА БОКХОВЕНА. А ПОСЛЕ ЕЕ СМЕРТИ - НА ЭЛИЗАБЕТ, ДОЧЕРИ ГОЛЛАНДЦА, ПОЛКОВНИКА РОНАЕРА. НИ ТОГО, НИ ДРУГОГО РОДА НЕТ В ЛЕКСИКОНЕ. МЫ НЕ ЗНАЕМ, ДОЖИЛ ЛИ РОД БОКХОВЕНОВ ДО XVIII В., НО ВОТ РОНАЕРЫ ДОЖИЛИ ПОЧТИ НАВЕРНЯКА, ИБО ПАТРИК ГОРДОН УМЕР В САМОМ КОНЦЕ 1699 Г., ОСТАВИВ ВДОВУ. Т.О., ОТСУТСТВИЕ ЭТОЙ СЕМЬИ ЯВЛЯЕТСЯ ЯВНЫМ УПУЩЕНИЕМ ЛЕКСИКОНА, ОПЯТЬ-ТАКИ, СВЯЗАННЫМ С НЕДОСТАТОЧНЫМ ИСПОЛЬЗОВАНИЕМ ИСТОРИОГРАФИИ И ПЕЧАТНЫХ ИСТОЧНИКОВ.

К ОЧЕНЬ СЕРЬЕЗНЫМ ДЕФЕКТАМ ЛЕКСИКОНА МЫ ВЫНУЖДЕНЫ ОТНЕСТИ ОТСУТСТВИЕ ИМЕННОГО УКАЗАТЕЛЯ. ЕСЛИ ДЛЯ СЛОВАРНОЙ ЧАСТИ КНИГИ ОН ИГРАЛ БЫ ЛИШЬ ВСПОМОГАТЕЛЬНУЮ РОЛЬ (ДЛЯ ИМЕН, УПОМИНАЕМЫХ ВНУТРИ СТАТЕЙ), ТО БЕЗ ТАКОГО УКАЗАТЕЛЯ МЕРТВЫМ БАЛЛАСТОМ ОКАЗЫВАЮТСЯ ПОЧТИ 50 СТРАНИЦ « ДОПОЛНЕНИЙ » (С. 237-283), СОДЕРЖАЩИЕ НЕСКОЛЬКО ТЫСЯЧ ИМЕН НАНЯТЫХ СПЕЦИАЛИСТОВ, ВЫЯВЛЕННЫХ ПО РАЗЛИЧНЫМ ЛИТЕРАТУРНЫМ ИСТОЧНИКАМ. РЕЦЕНЗЕНТ СДЕЛАЛ ПОПЫТКУ ПРОВЕРИТЬ ПО ЭТИМ СПИСКАМ НЕСКОЛЬКО ИНТЕРЕСОВАВШИХ ЕГО ИМЕН, НО БЫЛ ВЫНУЖДЕН ОТКАЗАТЬСЯ ОТ ЭТОЙ ИДЕИ. ЗАКЛЮЧЕНИЕ. ПЕРЕД НАМИ ПЕРВЫЙ РЕЗУЛЬТАТ МНОГОЛЕТНЕЙ И ФУНДАМЕНТАЛЬНОЙ РАБОТЫ, КОТОРАЯ ДОЛЖНА БЫТЬ ЗАВЕРШЕНА С ОСОБЫМ ВНИМАНИЕМ К ИСТОРИОГРАФИИ И ПЕЧАТНЫМ ИСТОЧНИКАМ, А ТАКЖЕ С ПОДГОТОВКОЙ ХОРОШЕГО СПРАВОЧНОГО АППАРАТА. 